\title{
Predição da distribuição diamétrica de uma floresta manejada experimentalmente através de um Sistema de Equações Diferenciais
}

\author{
Celso Paulo de AZEVEDOํ, Carlos Roberto SANQUETTA², José Natalino Macedo SILVA³, Sebastião Amaral \\ $\mathrm{MACHADO}^{4}$, Cíntia Rodrigues de SOUZA5, Milena Marmentine de OLIVEIRA ${ }^{6}$
}

\begin{abstract}
RESUMO
Este estudo teve como objetivo principal projetar a distribuição diamétrica da floresta manejada através de um sistema de equaçōes diferenciais de primeira ordem, ajustado para cada tratamento. Os dados básicos foram obtidos no Projeto Bom Manejo (Embrapa Amazônia Oriental/CIFOR/ITTO), na Companhia Florestal Monte Dourado (Jarí), Vitória do Jarí - AP. $\mathrm{O}$ delineamento é em blocos ao acaso com três repetições. Os tratamentos são combinaçōes de intensidades de exploração $(15 \%, 25 \%$ e $35 \%$ do volume total das árvores com mais de $60 \mathrm{~cm}$ de DAP) com intensidades de desbastes $(0 \%, 30 \%$, $50 \%$ e $70 \%$ da área basal original). Utilizou-se como controle a floresta não explorada. $\mathrm{Na}$ área experimental estão locadas 40 parcelas permanentes de 1,0 ha cada, sendo 36 exploradas e quatro não exploradas. O povoamento foi medido em 1984, explorado em 1985 e remedido em 1986, 1988, 1990, 1994, 1996 e 2004. Foram medidas todas as árvores com DAP $\geq 20$ $\mathrm{cm}$. Conquanto esse sistema de equações mantenha a simplicidade de abordagem do problema inerente aos modelos baseados em matrizes de transição, também apresenta sobre estes últimos a vantagem adicional de permitir a obtenção dos valores das variáveis de estado do sistema para qualquer ponto no tempo, não se restringindo a intervalos múltiplos do intervalo original usado na derivação da matriz de transição. Assim, o método foi constatado para diferentes períodos de avaliaçóes e os resultados mostraram que nem os períodos de projeções mais longos (ponto de equilíbrio) serão suficientes para restaurar as condiçōes iniciais do povoamento.
\end{abstract}

PALAVRAS-CHAVE: Manejo florestal, distribuição diamétrica, modelos de crescimento, dinâmica florestal, Amazônia.

\section{Diametric distribution prediction of an experimentally managed forest through a differential equation system}

\begin{abstract}
The aim of this study was to evaluate the prognosis of the diameter structure of an experimentally managed forest, utilizing a system of differential equations of the first order, adjusted for each treatment. The basic data were obtained from the Bom Manejo Projet (Embrapa Eastern Amazon/CIFOR/ITTO), carried out in the Monte Dourado Forest Company (Jarí) area, Vitória do Jari, Amapá, Brazil. The statistical delineation was designed as random blocks with three replications. The treatments applied were combinations of logging intensities $(15 \%, 25 \%$ and $35 \%$ of the total volume of the trees larger than $60 \mathrm{~cm}$ of $\mathrm{DBH})$ and thinning intensities $(0 \%, 30 \%, 50 \%$ and $70 \%$ of the original basal area). The non-logged forest was used for the control plots. Stand development was monitored through 40 permanent sample plots of 1 ha each (36 logged and 4 unlogged). The stand was measured in 1984, logged in 1985 and remeasured in 1986, 1988, 1990, 1994, 1996 and 2004. All the trees with $\mathrm{DBH} \geq 20 \mathrm{~cm}$ were measured. While keeping the same simplicity of transition matrix based models, the proposed model allows for determining the state of the system for any given point in time, not being confined just for multiplying time intervals as was the former. This method was verified for different evaluation periods and the results showed that not even the longer periods of projections (break-even point) will be enough to restore the initial conditions of the stand.
\end{abstract}

KEY WORDS: Forest management, diametric distribution, growth models, forest dynamics, Amazon.

1 Engenheiro Florestal, Doutor, Pesquisador, Embrapa Amazônia Ocidental, km 29 da AM 010, Caixa Postal 319, 69011-970, Manaus - AM. e-mail: celso@cpaa.embrapa.br

2 Engenheiro Florestal, Doutor, Professor Adjunto, Universidade Federal do Paraná, Departamento de Ciências Florestais, Av. Lothário Meissner, 3400 - Jardim Botânico - Campus III, 80210-170, Curitiba - PR. e-mail: sanqueta@floresta.ufpr.br

${ }^{3}$ Engenheiro Florestal, Doutor, Pesquisador, Embrapa Amazônia Oriental, Trav. Dr. Enéias Pinheiro s/n, Caixa Postal 48, Bairro do Marco, 66095-100, Belém - PA. e-mail: natalino. silva@cgiar.org

${ }^{4}$ Engenheiro Florestal, PhD., Professor Titular, Universidade Federal do Paraná, Departamento de Ciências Florestais, Pesquisador 1 A do CNPq, Av. Lothário Meissner, 3400 - Jardim Botânico - Campus III, 80210-170, Curitiba - PR. e-mail: sammac@floresta.ufpr.br

${ }^{5}$ Engenheira Florestal, MSc., Pesquisadora, Embrapa Amazônia Ocidental, km 29 da AM 010, Caixa Postal 319, 69011-970, Manaus - AM. e-mail: cintia@cpaa.embrapa.br

${ }^{6}$ Estudante de Engenharia Florestal, Bolsista PET, Estagiária LIF, Universidade Federal do Paraná, Departamento de Ciências Florestais, Av. Lothário Meissner, 3400 - Jardim Botânico - Campus III, 80210-170, Curitiba - PR. e-mail: mifloresta@ufpr.br 


\section{INTRODUÇÃO}

Os modelos de crescimento podem ser divididos em três categorias: (1) modelos de povoamento total, (2) modelos de distribuição por classe de tamanho e (3) modelos de árvores individuais (Daniels \& Burkhart, 1988).

Modelos do tipo povoamento total fornecem estimativas do crescimento e/ou da produção para o povoamento como um todo (ou para porçôes comerciais). Usualmente, esses modelos são construídos mediante o uso de técnicas de regressōes múltiplas, como as desenvolvidas por Clutter (1963) e Sullivan \& Clutter (1972).

Modelos do tipo árvores individuais requerem particularidade de cada árvore no povoamento. Segundo Botkin et al. (1972), esses tipos de modelos apresentam as seguintes características: cada árvore é definida por variáveis, como: idade, diâmetro e altura máxima, relação entre altura e diâmetro, taxa de fotossíntese, necessidade de luz, relações entre crescimento relativo e uma medida de clima. Outras variáveis ambientais não-bióticas, como profundidade do solo, capacidade de armazenamento de água no solo, percentagem de rocha no solo, média mensal de temperatura e precipitação e insolação anual acima da copa da floresta.

As espécies são também diferenciadas por probabilidades de sobrevivência e por adições de novas plantas. Munro (1974), citado por Daniels \& Burkhart (1988), dividiu esses modelos em duas classes: modelos do tipo distância dependente e do tipo distância independente, caso a localização individual das árvores seja requerida, ou não, como atributo do modelo.

Os modelos de classe de tamanho são intermediários entre os modelos de povoamento total e os modelos de árvores individuais. Os modelos de classe de tamanho incluem projeção de tabelas de povoamento, matrizes de transição e modelos de "cohort" (Vanclay, 1989; Alder, 1995).

Entre os modelos de matrizes de transição, a cadeia de Markov foi utilizada por vários pesquisadores na prognose e no desenvolvimento de povoamentos ineqüiâneos, como: Buongiorno \& Michie (1980), Higuchi (1987), Azevedo (1993), Sanquetta et al. (1996), dentre outros.

O objetivo deste trabalho foi simular o efeito de diferentes níveis de exploração e tratamentos silviculturais sobre a dinâmica de crescimento da floresta remanescente. A evolução da distribuição diamétrica foi adequadamente formulada e resolvida como um sistema de equações diferenciais de primeira ordem. A solução do sistema de equações diferenciais conduziu a equações individuais que descrevem o número de árvores em cada classe diamétrica como uma função do tempo. Os níveis de exploração foram estudados como cenários de manejo, mantendo-se a remoção periódica do número de árvores nas diversas classes de diâmetro. Fez-se coincidir os períodos de projeção com os de avaliações.

\section{MATERIAL E MÉTODOS}

\section{LOCALIZAÇÃO E CARACTERIZAÇÃO DA ÁREA}

Este estudo foi conduzido com dados do Projeto Bom Manejo (Embrapa/CIFOR/ITTO) - Manejo sustentável de florestas de produção em escala comercial na Amazônia brasileira, desenvolvido pela Embrapa Amazônia Oriental. O experimento foi iniciado em 1983, em uma área de 500 ha na Companhia Florestal Monte Dourado (Jari), na localidade Morro do Felipe, Vitória do Jari - AP, coordenada $52^{\circ} 20^{\prime \prime}$ W e $00^{0} 55^{\prime}$ "S, a uma altitude de aproximadamente $150 \mathrm{~m}$. O clima nessa região é do tipo Ami, pela classificação de Köppen. A precipitação média anual alcança $2.234 \mathrm{~mm}$. A temperatura média anual é de $25,8^{\circ} \mathrm{C}$, a vegetação é do tipo Floresta Ombrófila Densa. Os solos são do tipo Latossolo Amarelo Distrófico, com textura argilosa pesada.

\section{DELINEAMENTO EXPERIMENTAL}

O delineamento foi estruturado em blocos ao acaso com três repetiçōes. As alternativas de manejo testadas foram combinaçôes de intensidades de exploração com intensidades de desbastes.

As intensidades de exploração foram representadas por porcentagens do volume extraído em relação ao volume total da floresta a partir do diâmetro à altura do peito (DAP) de 60 $\mathrm{cm}$. Aplicaram-se intensidades de 15\% (leve), 25\% (média) e $35 \%$ (pesada). Essas foram combinadas com quatro tipos de intensidades de desbastes, $0 \%, 30 \%, 50 \%$ e $70 \%$ de redução da área basal original, o desbaste sistemático, que consiste em desvitalizar árvores não comerciais a partir de um diâmetro mínimo selecionado até atingir a redução de área basal planejada e, o desbaste seletivo ou de liberação de copas, que consiste em desvitalizar árvores de espécies nãocomerciais cujas copas estavam competindo por luz com as copas das árvores selecionadas para a próxima colheita (Tabela 1). A exploração foi feita em faixas de $200 \mathrm{~m}$ x $800 \mathrm{~m}$. Fora dos blocos a intensidade de exploração foi de $35 \%$ do volume (Figura 1).

\section{MONITORAMENTO DA FLORESTA}

$\mathrm{Na}$ área experimental estão locadas 40 parcelas permanentes de 1 hectare, sendo 36 na área explorada e quatro na área não explorada. A primeira medição foi realizada em 1984, a exploração em 1985 e as remediçôes em 1986, 1988, 1990, 1994,1996 e 2004. Todas as árvores com diâmetro maior ou igual a $20,0 \mathrm{~cm}$ foram medidas periodicamente nas parcelas. A metodologia empregada para instalação e coleta de dados das unidades amostrais (parcelas permanentes) foi desenvolvida e disponibilizada por Silva \& Lopes (1984). 


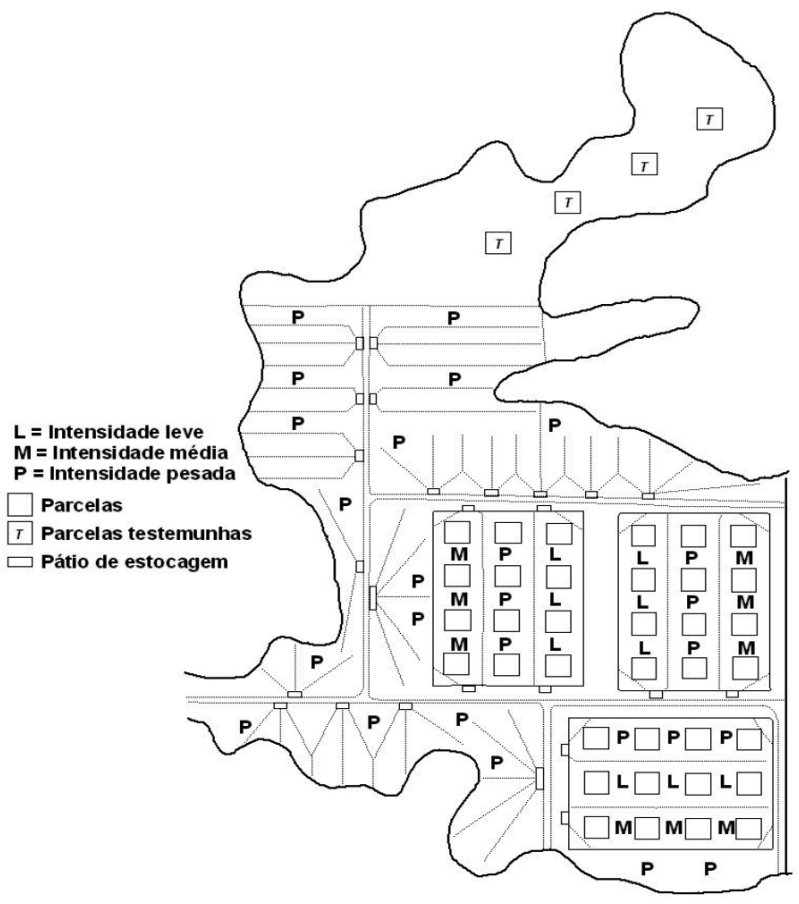

Figura 1 - Croqui do experimento ( $\mathrm{L}$ - intensidade leve; $\mathrm{M}$ - intensidade média; $\mathrm{P}$ - intensidade pesada).

\section{EQUAÇÕES DIFERENCIAIS DE PRIMEIRA ORDEM}

A estrutura da distribuição diamétrica do povoamento foi estudada sob a forma de equaçóes diferenciais de primeira ordem, para cada tratamento. Esse sistema de equações permite obter os valores das variáveis de estado do sistema para qualquer ponto no tempo, não se restringindo a intervalos múltiplos do intervalo original na derivação da matriz de transição.

\section{FORMULAÇÃO E SOLUÇÃO DO MODELO}

A formulação do modelo correspondente baseia-se na estratégia adotada no modelo matricial de Buongiorno \& Michie (1980).

Sejam as seguintes variáveis:

$Y_{i}(t): \mathrm{n}^{\circ}$, por unidade de área, de árvores vivas na i-ésima classe diamétrica, no tempo t;

$B(t)$ : área basal total por unidade de área, no tempo t;

$N(t)$ : número total, por unidade de área, de árvores vivas no tempo t.

Após testar numerosas variáveis e combinaçōes de variáveis propostas por Moser (1972), Solomon et al. (1986) e Davidson et al. (1989), a melhor regressão para o ingresso foi dada pela seguinte expressão:

$$
Y_{0}(t)=\beta_{0}-\beta_{1} B(t)+\beta_{2} N(t)+\beta_{3} \Delta_{t}
$$

Tabela 1 - Descrição dos tratamentos.

\begin{tabular}{|c|c|}
\hline Tratamento & Descrição \\
\hline $\mathrm{T}_{1}$ & $\begin{array}{l}\text { - Exploração de 15\% do volume total das árvores de DAP > } \\
60 \mathrm{~cm} \text {, sem redução da área basal após a exploração; }\end{array}$ \\
\hline $\mathrm{T}_{2}$ & $\begin{array}{l}\text { - Exploração de } 15 \% \text { do volume total das árvores de DAP > } \\
60 \mathrm{~cm} \text {, com redução de } 30 \% \text { da área basal original através } \\
\text { de tratos silviculturais; }\end{array}$ \\
\hline $\mathrm{T}_{3}$ & $\begin{array}{l}\text { - Exploração de } 15 \% \text { do volume total das árvores de DAP > } \\
60 \mathrm{~cm} \text {, com redução de } 50 \% \text { da área basal original através } \\
\text { de tratos silviculturais; }\end{array}$ \\
\hline $\mathrm{T}_{4}$ & $\begin{array}{l}\text { - Exploração de } 15 \% \text { do volume total das árvores de DAP > } \\
60 \mathrm{~cm} \text {, com redução de } 70 \% \text { da área basal original através } \\
\text { de tratos silviculturais; }\end{array}$ \\
\hline $\mathrm{T}_{5}$ & $\begin{array}{l}\text { - Exploração de } 25 \% \text { do volume total das árvores de DAP > } \\
60 \mathrm{~cm} \text {, sem redução da área basal após a exploração; }\end{array}$ \\
\hline $\mathrm{T}_{6}$ & $\begin{array}{l}\text { - Exploração de } 25 \% \text { do volume total das árvores de DAP > } \\
60 \mathrm{~cm} \text {, com redução de } 30 \% \text { da área basal original através } \\
\text { de tratos silviculturais; }\end{array}$ \\
\hline $\mathrm{T}_{7}$ & $\begin{array}{l}\text { - Exploração de } 25 \% \text { do volume total das árvores de DAP > } \\
60 \mathrm{~cm} \text {, com redução de } 50 \% \text { da área basal original através } \\
\text { de tratos silviculturais; }\end{array}$ \\
\hline $\mathrm{T}_{8}$ & $\begin{array}{l}\text { - Exploração de } 25 \% \text { do volume total das árvores de DAP > } \\
60 \mathrm{~cm} \text {, com redução de } 70 \% \text { da área basal original através } \\
\text { de tratos silviculturais; }\end{array}$ \\
\hline $\mathrm{T}_{9}$ & $\begin{array}{l}\text { - Exploração de 35\% do volume total das árvores de DAP > } \\
60 \mathrm{~cm} \text {, sem redução da área basal após a exploração; }\end{array}$ \\
\hline $\mathrm{T}_{10}$ & $\begin{array}{l}\text { - Exploração de } 35 \% \text { do volume total das árvores de DAP > } \\
60 \mathrm{~cm} \text {, com redução de } 30 \% \text { da área basal original através } \\
\text { de tratos silviculturais. }\end{array}$ \\
\hline $\mathrm{T}_{11}$ & $\begin{array}{l}\text { - Exploração de } 35 \% \text { do volume total das árvores de DAP > } \\
60 \mathrm{~cm} \text {, com redução de } 50 \% \text { da área basal original através } \\
\text { de tratos silviculturais; }\end{array}$ \\
\hline $\mathrm{T}_{12}$ & $\begin{array}{l}\text { - Exploração de } 35 \% \text { do volume total das árvores de DAP > } \\
60 \mathrm{~cm} \text {, com redução de } 70 \% \text { da área basal original através } \\
\text { de tratos silviculturais. }\end{array}$ \\
\hline
\end{tabular}

Onde $\beta_{0}, \beta_{1}, \beta_{2}$ e $\beta_{3}$ são constantes positivas e $\Delta_{t}$ é o intervalo de tempo entre duas medições.

A área basal total, por unidade de área, no tempo t, foi calculada pela seguinte fórmula:

$$
B(t)=\sum_{i=1}^{n} \frac{\pi d_{i}^{2}}{4} Y_{i}(t)
$$

onde $\mathrm{d}_{\mathrm{i}}$ é o diâmetro no centro da i-ésima classe diamétrica.

O número total de árvores, por unidade de área, no tempo t, foi dado por:

$$
N(t)=\sum_{i=1}^{n} Y_{i}(t)
$$

Substituindo-se as equações 2 e 3 em 1, tem-se: 


$$
Y_{0}(t)=\beta_{0}-\sum_{i=1}^{n}\left[\left(\beta_{1} \frac{\pi d_{i}^{2}}{4}-\beta_{2}\right) Y_{i}(t)\right]+\beta_{3} \Delta_{t}
$$

A variação no número de árvores da i-ésima classe diamétrica, durante um intervalo de tempo, é igual ao número de árvores que ingressaram nessa classe, menos o número de árvores que deixaram a i-ésima classe. Essa variação foi expressa, sob a forma de uma equação diferencial, como se segue:

$$
\frac{d Y_{i}}{d t}=a_{i 1} Y_{i-1}-\left(a_{i 2}+a_{i 3}\right) Y_{i}+i, \text { para } \mathrm{i}=1,2, \ldots, \mathrm{n}
$$

Onde:

$a_{i 1}=$ porcentagem de árvores movendo-se da classe diamétrica " -1 ” para a i-ésima classe diamétrica $\left(0<a_{i 1} \leq\right.$ $1 \forall i \leq n)$;

$a_{i 2}=$ porcentagem de árvores movendo-se da i-ésima classe diamétrica para a classe imediatamente superior $\left(0<a_{i 2} \leq 1\right.$ $\forall i \leq n$ e $a_{n 2}=0$ );

$a_{i 3}=$ porcentagem de mortalidade de árvores da i-ésima classe diamétrica durante $\mathrm{dt}\left(0<a_{i 3} \leq 1 \forall i \leq n\right)$;

$b_{i}=$ constante associada à i-ésima classe diamétrica $\left(b_{i}=\right.$ $\beta_{0}+\beta_{3} \Delta_{t}$ para $i=1$ e $b_{i}=0 \forall i>1$ ).

A descrição matemática da taxa de variação do número de árvores para cada uma das $\mathrm{n}$ classes de diâmetro produzirá o seguinte sistema genérico de equaçôes diferenciais de primeira ordem:

$$
\begin{gathered}
\frac{d Y_{1}}{d t}=a_{11} Y_{0}-\left(a_{12}+a_{13}\right) Y_{1}+\beta_{0}+\beta_{3} \Delta_{t} \\
\frac{d Y_{2}}{d t}=a_{21} Y_{1}-\left(a_{22}+a_{23}\right) Y_{2}+0 \\
\frac{d Y_{3}}{d t}=a_{31} Y_{2}-\left(a_{32}+a_{33}\right) Y_{3}+0 \\
\bullet \\
\bullet \\
\frac{d Y_{n}}{d t}=a_{n 1} Y_{n-1}-a_{n 3} Y_{n}+0
\end{gathered}
$$

Utilizando a notação matricial esse sistema pode ser escrito como:

$$
\bar{Y}^{\prime}=A \cdot \bar{Y}+\bar{b}
$$

Para o caso homogêneo, tem-se que $\bar{b}=\overline{0}$ e a equação 6 se resume a:

$$
\bar{Y}^{\prime}=A \cdot \bar{Y}
$$

Se a matriz A possui $n$ autovetores $\bar{\rho}_{\lambda_{1}}, \ldots, \bar{\rho}_{\lambda_{n}}$ associados aos respectivos autovalores, $\lambda_{1}, \ldots, \lambda_{\mathrm{n}}$, então a solução geral de [7] será dada por Zill \& Cullen (2001):

$$
\bar{Y}=\bar{\rho}_{\lambda_{1}} \cdot e^{\lambda_{1} t}=\ldots=\bar{\rho}_{\lambda_{n}} \cdot e^{\lambda_{n} t} \Rightarrow \bar{Y}=\bar{\rho}_{\lambda} \cdot \epsilon
$$

Substituindo a equação 8 na equação 7 , tem-se a equação 9.

$$
\bar{Y}^{\prime}=A \cdot \bar{\rho}_{\lambda} \cdot e^{\lambda t}
$$

Tomando a derivada da equação 8 em relação a " $\mathrm{t}$ ", obtémse a equação 10 .

$$
\frac{d \bar{Y}}{t}=\overline{Y^{\prime}}=\lambda \cdot \bar{\rho}_{\lambda} \cdot e^{\lambda t}=\lambda \cdot I_{n} \cdot \bar{\rho}_{\lambda} \cdot e^{\lambda t}
$$

em que $\mathrm{I}_{\mathrm{n}}$ é a matriz identidade de ordem $n$.

Subtraindo a equação 10 da equação 9 , tem-se:

$$
A \cdot \bar{\rho}_{\lambda} \cdot e^{\lambda t}-\lambda \cdot I_{n} \cdot \bar{\rho}_{\lambda} \cdot e^{\lambda t}=\overline{0} \Rightarrow\left[A-\lambda \cdot I_{n}\right] \bar{\rho}_{\lambda} \cdot e^{\lambda t}=\overline{0}
$$

Dado que $e^{\lambda t} \neq 0$, então a equação 11 somente será satisfeita se:

$$
\left[A-\lambda . I_{n}\right] \bar{\rho}_{\lambda}=\overline{0}
$$

Por outro lado, o sistema de equação terá solução não trivial, isto é, não nula, para $\bar{\rho}_{\lambda}$ se, e somente se, a seguinte condição for verificada.

$$
\operatorname{det}\left(A-\lambda \cdot I_{n}\right)=\overline{0}
$$

A obtenção do conjunto de autovalores, $\lambda_{1}, \ldots, \lambda_{\mathrm{n}}$, requer solução da equação 13 . Especificamente, $\bar{\rho}_{\lambda_{k}}$ é dito ser um autovalor de $\mathbf{A}$ se a seguinte condiçāo for satisfeita:

$$
\left(A-\lambda . I_{n}\right) \bar{\rho}_{\lambda_{k}}=\overline{0}
$$

em que $\left(\bar{\rho}_{\lambda_{k}}\right)^{\prime}=\left[\rho_{1}, \ldots, \rho_{n}\right]$

$\mathrm{O}$ autovalor $\bar{\rho}_{\lambda_{k}}$ será obtido, substituindo $\lambda_{\mathrm{k}}$ na equação 12. Uma vez que os autovetores $\bar{\rho}_{\lambda_{1}}, \ldots, \bar{\rho}_{\lambda_{n}}$ tenham sido determinados e se todos os autovalores $\lambda_{1}, \ldots, \lambda_{\mathrm{n}}$, possuírem multiplicidades igual a 1 , isto é, se autovalor ocorrer uma única vez como solução da equação 13 , então a solução homogênea do sistema de equaçóes 6 será dada (Zill \& Cullen, 2001) por:

$$
\bar{Y}(t)=\sum_{i=1}^{n}\left(e^{\lambda_{i} t} \cdot \bar{\rho}_{\lambda_{i}} \cdot c_{i}\right)
$$

em que $c_{\mathrm{i}}$ são constantes estabelecidas pelas condições iniciais.

Define-se agora $\bar{w}(t)$ como sendo a matriz fundamental de solução para o sistema de equação 7 , da seguinte forma:

$$
\bar{w}(t)=e^{A t}=\left[\bar{w}_{1}(t), \ldots, \bar{w}_{n}(t)\right]
$$

em que $\overline{w_{i}}(t)=e^{\lambda_{i} t} \cdot \bar{\rho}_{\lambda_{i}}$ é a i-ésima coluna da matriz $\bar{w}(t)$, sendo, portanto, o i-ésimo vetor solução. É conveniente agrupar as 
constantes $c_{i}$ da equação 15 em um vetor linha, da seguinte forma $(\bar{c})^{T}=\left[c_{1}, \ldots, c_{n}\right]$.

Assim, a equação 15 poderá ser reescrita da seguinte maneira:

$$
\bar{Y}(t)=\bar{w}(t) \bar{c}
$$

A equação 17 é a solução homogênea, sob notação matricial, para o sistema de equações diferenciais de primeira ordem, dado pela equação 6 .

Para o caso de um sistema não homogêneo $(\bar{b} \neq \overline{0})$, a solução particular da equação 6 será dada por:

$$
A \cdot \bar{Y}=-\bar{b} \Rightarrow \bar{Y}=-A^{-1} \cdot \bar{b}
$$

A solução geral desse sistema poderá ser, então, obtida pela soma das equações 17 e 18, o que leva a:

$$
\bar{Y}(t)=\bar{w}(t) \bar{c}-A^{-1} \cdot \bar{b}
$$

\section{RESULTADOS E DISCUSSÃO}

\section{INGRESSO}

Testaram-se vários modelos, combinando-se ou não as variáveis independentes, área basal e número de árvores por hectare (Moser, 1972; Ek, 1974; Davidson et al., 1989; Buongiorno \& Michie, 1980), considerando-se a possibilidade de utilização de uma ou outra, ou ambas, na estimativa do ingresso por hectare.

De acordo com as estatísticas apresentadas, o modelo que proporciona maior precisão na estimativa do ingresso, foi dado pela equação a seguir, com a inclusão da variável intervalo de tempo $\left(\Delta_{\mathrm{T}}\right)$ entre as mediçóes.

$$
\begin{aligned}
& Y_{\theta}(t)=9,27484-0,467592 G-0,00804854 N+4,14433 \Delta_{T} \\
& R_{\text {ajustado }}^{2}=78,30 \% \quad S_{y x}(\%)=43,52 \quad F(3,230)=281,19 \quad(p=0,0000)
\end{aligned}
$$

A análise desta equação permite concluir que o ingresso será maior em povoamentos com menor número de árvores - essas com menor diâmetro - e quanto maior for o intervalo de tempo entre as medições. Todos os coeficientes foram significativos a $1 \%$ de probabilidade.

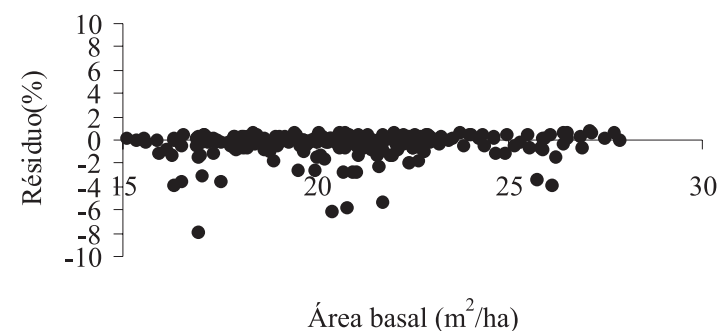

Figura 2 - Distribuição gráfica dos resíduos para o modelo de ingresso.
$\mathrm{Na}$ Figura 2, observa-se a distribuição gráfica dos resíduos para a equação acima. A equação apresenta resíduos próximos à linha de nulidade e bem distribuídos.

\section{PROJEÇÃO DA DISTRIBUIÇÃO DIAMÉTRICA}

Os elementos da matriz de transição, para cada tratamento, foram determinados de acordo com Buongiorno \& Michie (1980), com base nos dados observados em duas ocasiōes diferentes (1984 e 1990). A matriz de transição foi estruturada considerando oito estados: estados 1 a 7 , classes diamétricas com intervalos de $10 \mathrm{~cm}$ e estado 8 , mortalidade (M). A transição de um estado i para outro (estado j) significa o seguinte: uma árvore medida em 1984 (n $\mathrm{n}_{\mathrm{i}}$ ) pode, em 1990, permanecer na mesma classe de diâmetro ou estado, mudar para uma ou mais classes seguintes ou morrer. A última classe diamétrica foi generalizada para DAP $\geq 80 \mathrm{~cm}$; assim, no estado 7 , os diâmetros podem variar de $80 \mathrm{~cm}$ até o maior diâmetro medido.

A Tabela 2 apresenta a transição, do ponto de vista absoluto, de um estado para outro, ocorrida durante o período de 1984 a 1990, usando todas as observaçōes das três repetições, 3 hectares, do Tratamento 4, que será apresentado em detalhes para exemplificar o uso do modelo.

A Tabela 3 apresenta a matriz de transição probabilística, criada a partir da Tabela 2. Agora, em bases probabilísticas, é mais fácil observar as chances de cada árvore em cada estado, seja para permanecer na mesma classe, mudar para uma ou mais classes adiante, ou morrer. De um modo geral, as árvores têm maiores probabilidades de permanecerem na mesma classe, principalmente considerando um período de apenas 6 anos. Em relação ao estado $\mathrm{M}$ (mortalidade) não é fácil definir um padrão. Este comportamento estocástico é típico da floresta tropical úmida, dificultando os trabalhos de modelagem da dinâmica da floresta, principalmente quando se usa modelos determinísticos. O recrutamento - quarenta e seis indivíduos - (100\%) aconteceu na primeira classe de diâmetro $(20 \mathrm{~cm} \leq \mathrm{DAP}<30 \mathrm{~cm})$.

Tabela 2 - Número de árvores que mudaram do estado i para 0 estado j, durante 0 intervalo de 6 anos, no tratamento 4.

\begin{tabular}{llllllll}
\hline \multirow{2}{*}{ ESTADO $\mathrm{j}$} & \multicolumn{2}{l}{ ESTADO $\mathrm{i}$} & \multicolumn{7}{l}{$\mathrm{l}$} \\
\cline { 2 - 8 } & 25 & 35 & 45 & 55 & 65 & 75 & 80 \\
\hline 25 & 209 & 0 & 0 & 0 & 0 & 0 & 0 \\
35 & 27 & 105 & 0 & 0 & 0 & 0 & 0 \\
45 & 0 & 14 & 55 & 0 & 0 & 0 & 0 \\
55 & 0 & 0 & 11 & 29 & 0 & 0 & 0 \\
65 & 0 & 0 & 1 & 2 & 9 & 0 & 0 \\
75 & 0 & 0 & 0 & 0 & 3 & 5 & 0 \\
$\geq 80$ & 0 & 0 & 0 & 0 & 0 & 2 & 8 \\
$\mathrm{M}$ & 42 & 13 & 4 & 4 & 1 & 4 & 8 \\
$\mathrm{n}_{\mathrm{i}}$ & 278 & 132 & 71 & 35 & 13 & 11 & 16 \\
\hline & & & & & & &
\end{tabular}


Tabela 4 - Sumarização da dinâmica da população do Tratamento 4.

\begin{tabular}{llllllll}
\hline $\begin{array}{l}\text { Classe } \\
\text { DAP }\end{array}$ & $\begin{array}{l}\mathrm{di} \\
(\mathrm{cm})\end{array}$ & $\begin{array}{l}\mathrm{Gi} \\
\left(\mathrm{m}^{2}\right)\end{array}$ & $\begin{array}{l}\text { Yi } \\
\left(\mathrm{n} . \mathrm{ha}^{-1}\right)\end{array}$ & $\begin{array}{l}\text { Colhidas }\left(\mathrm{h}_{\mathrm{i}}\right) \\
\left(\mathrm{n} \cdot \mathrm{ha}^{-1}\right)\end{array}$ & $\begin{array}{l}\text { Yif } \\
\left(\mathrm{Yi}-\mathrm{h}_{\mathrm{i}}\right)\end{array}$ & $\begin{array}{l}\text { Taxa de Migração } \\
\text { para a Classe seguinte } \\
\text { de DAP (decimal) }\end{array}$ & $\begin{array}{l}\text { Mortalidade } \\
(\text { decimal) }\end{array}$ \\
\hline $20-29,9 \mathrm{~cm}$ & 25 & 0,0491 & 92,67 & 0,00 & 92,67 & 0,10 & 0,15 \\
$30-39,9 \mathrm{~cm}$ & 35 & 0,0962 & 44,00 & 0,00 & 44,00 & 0,11 & 0,10 \\
$40-49,9 \mathrm{~cm}$ & 45 & 0,1590 & 23,67 & 0,33 & 23,33 & 0,17 & 0,06 \\
$50-59,9 \mathrm{~cm}$ & 55 & 0,2376 & 11,67 & 0,00 & 11,67 & 0,06 & 0,11 \\
$60-69,9 \mathrm{~cm}$ & 65 & 0,3318 & 4,33 & 1,00 & 3,33 & 0,23 & 0,08 \\
$70-79,9 \mathrm{~cm}$ & 75 & 0,4418 & 3,67 & 1,00 & 2,67 & 0,18 & 0,36 \\
$\geq 80 \mathrm{~cm}$ & 80 & 0,5027 & 5,33 & 0,67 & 4,67 & 0,00 & 0,50 \\
\hline
\end{tabular}

Tabela 3 - Matriz de transição probabilística do estado $\boldsymbol{i}$ para 0 estado $\boldsymbol{j}$, durante o intervalo de 6 anos, no tratamento 4.

\begin{tabular}{llllllll}
\hline \multirow{2}{*}{ ESTADO } & & \multicolumn{6}{l}{ ESTADO $\mathrm{i}$} \\
\cline { 2 - 8 } & 25 & 35 & 45 & 55 & 65 & 75 & $\geq 80$ \\
\hline 25 & 0,7518 & 0 & 0 & 0 & 0 & 0 & 0 \\
35 & 0,0971 & 0,7955 & 0 & 0 & 0 & 0 & 0 \\
45 & 0 & 0,1061 & 0,7746 & 0 & 0 & 0 & 0 \\
55 & 0 & 0 & 0,1690 & 0,8286 & 0 & 0 & 0 \\
65 & 0 & 0 & 0 & 0,0571 & 0,6923 & 0 & 0 \\
75 & 0 & 0 & 0 & 0 & 0,2308 & 0,4545 & 0 \\
$\geq 80$ & 0 & 0 & 0 & 0 & 0 & 0,1818 & 0,5000 \\
$M$ & 0,1511 & 0,0985 & 0,0563 & 0,1143 & 0,0769 & 0,3636 & 0,5000 \\
\hline
\end{tabular}

A dinâmica da população do Tratamento 4 está sumarizada na Tabela 4. Utilizando-se os dados da Tabela 4, o sistema de equaçôes diferenciais que descreve a variação do número de árvores em cada classe de DAP ao longo do tempo pode ser formulado como segue:

$$
\begin{aligned}
& \frac{d Y_{1}}{d t}=Y_{0}-(0,10+0,15) Y_{1} \\
& \frac{d Y_{2}}{d t}=0,10 Y_{1}-(0,11+0,10) Y_{2} \\
& \frac{d Y_{3}}{d t}=0,11 Y_{2}-(0,17+0,06) Y_{3} \\
& \frac{d Y_{4}}{d t}=0,15 Y_{3}-(0,06+0,11) Y_{4} \\
& \frac{d Y_{5}}{d t}=0,06 Y_{4}-(0,23+0,08) Y_{5} \\
& \frac{d Y_{6}}{d t}=0,23 Y_{5}-(0,18+0,36) Y_{6} \\
& \frac{d Y_{7}}{d t}=0,18 Y_{6}-0,50 Y_{7}
\end{aligned}
$$

Reescrevendo a equação de ingresso em termos do número de árvores em cada classe de DAP, tem-se:

\footnotetext{
$Y_{0}=9,27484-0,467592\left(0,0491 Y_{1}+0,0962 Y_{2}+0,1590 Y_{3}+0,2376 Y_{4}+0,3318 Y_{5}+0,4418 Y_{6}\right.$ $\left.+0,5027 Y_{7}\right)-0,00804854\left(Y_{1}+Y_{2}+Y_{3}+Y_{4}+Y_{5}+Y_{6}+Y_{7}\right)+4,14433(5,92)$

$Y_{0}=33,80-0,031001 Y_{1}-0,053036 Y_{2}-0,082416 Y_{3}-0,119140 Y_{4}-0,163210 Y_{5}-0,214624 Y_{6}$ $-0,243086 Y$
}

Substituindo $Y_{0}$ na primeira equação do sistema de equações diferenciais, tem-se:

$$
\begin{aligned}
\frac{d Y_{1}}{d t} & =33,80-0,281001 Y_{1}-0,053036 Y_{2}-0,082416 Y_{3}-0,119140 Y_{4}-0,163210 Y_{5}-0,214624 Y_{6} \\
& -0,243086 Y_{7} \\
\frac{d Y_{2}}{d t} & =0,10 Y_{1}-0,21 Y_{2} \\
\frac{d Y_{3}}{d t} & =0,11 Y_{2}-0,23 Y_{3} \\
\frac{d Y_{4}}{d t} & =0,15 Y_{3}-0,17 Y_{4} \\
\frac{d Y_{5}}{d t} & =0,06 Y_{4}-0,31 Y_{5} \\
\frac{d Y_{6}}{d t} & =0,23 Y_{5}-0,54 Y_{6} \\
\frac{d Y_{7}}{d t} & =0,18 Y_{6}-0,50 Y_{7}
\end{aligned}
$$

Utilizando-se a notação matricial, esse conjunto de equações poderá ser escrito como $\overline{Y^{\prime}}=A \bar{Y}+\bar{b}$ em que

$$
\begin{aligned}
& A=\left[\begin{array}{rrrrrrr}
-0,28100 & -0,053036 & -0,082416 & -0,11914 & -0,16321 & -0,214624 & -0,243085 \\
0,10 & -0,21 & 0 & 0 & 0 & 0 & 0 \\
0 & 0,11 & -0,23 & 0 & 0 & 0 & 0 \\
0 & 0 & 0,15 & -0,17 & 0 & 0 & 0 \\
0 & 0 & 0 & 0,06 & -0,31 & 0 & 0 \\
0 & 0 & 0 & 0 & 0,23 & -0,54 & 0 \\
0 & 0 & 0 & 0 & 0 & 0,18 & -050
\end{array}\right] \\
& \bar{Y}=\left[\begin{array}{l}
Y_{1 f} \\
Y_{2 f} \\
Y_{3 f} \\
Y_{4 f} \\
Y_{5 f} \\
Y_{6 f} \\
Y_{7 f}
\end{array}\right]
\end{aligned}
$$


A determinação dos autovalores do sistema de equações diferenciais é feita desenvolvendo-se a equação 13. Para o presente estudo existem sete valores distintos de $\lambda$ que satisfazem o sistema de equações.

A próxima etapa refere-se à determinação dos respectivos autovetores, que é feita mediante a aplicação da equação 12 . Como a partir deste ponto as soluçōes são muito complexas, recomenda-se acompanhar as soluções apresentadas por Ribeiro (1996), Zill e Cullen (2001) e Zill (2003) que, para simplificar a formulação e análise do modelo, apresentaram soluçôes para a matriz real $\boldsymbol{A} 2 \times 2$. O primeiro autor agrupou as árvores do povoamento em somente duas classes diamétricas.

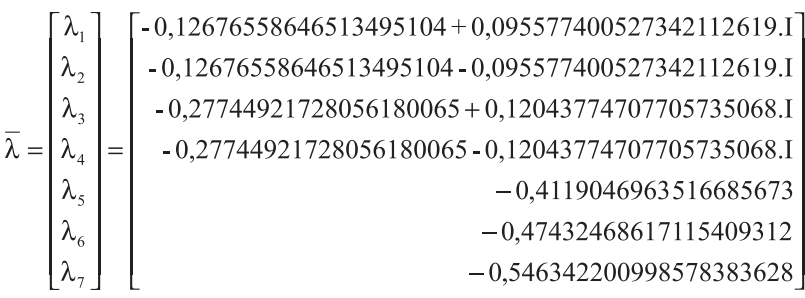

Os vetores soluçóes do sistema foram obtidos pela aplicação da equação 16 e a solução homogênea para o sistema foi obtida empregando-se a equação 17. Com a aplicação da equação 18 , temos a solução particular do sistema de equações diferenciais que representa os valores de equilíbrio, isto é, o número-limite de árvores nas respectivas classes diamétricas. Para o Tratamento 4, a situação de equilíbrio é dada por:

Tabela 5 - Evolução no número de árvores observado $\left(\mathrm{y}_{\mathrm{i}}\right)$ e projetado $\left(Y_{i}\right)$ em 1984, 1986, 1988, 1990, 1994, 1996 e 2004, por hectare, por tratamento e por classe de diâmetro.

\begin{tabular}{|c|c|c|c|c|c|c|c|c|c|c|c|c|c|c|c|c|c|c|}
\hline \multirow{2}{*}{ Trat } & \multirow{2}{*}{$\begin{array}{l}\mathscr{W} \\
\mathbb{N} \\
\mathbb{N} \\
\mathbb{T} \\
\end{array}$} & \multicolumn{4}{|c|}{1984} & \multicolumn{2}{|c|}{1986} & \multicolumn{2}{|c|}{1988} & \multicolumn{2}{|c|}{1990} & \multicolumn{2}{|c|}{1994} & \multicolumn{2}{|c|}{1996} & \multicolumn{2}{|c|}{2004} & \multirow{2}{*}{$\begin{array}{l}\text { 음 } \\
\text { 言 } \\
\text { 山्u }\end{array}$} \\
\hline & & $Y_{i}$ & $h_{i}$ & $Y_{\text {if }}$ & $\hat{Y}_{i}$ & $Y_{i}$ & $\hat{Y}_{i}$ & $Y_{i}$ & $\hat{Y}_{i}$ & $Y_{i}$ & $\hat{Y}_{i}$ & $Y_{i}$ & $\hat{Y}_{i}$ & $Y_{i}$ & $\hat{Y}_{i}$ & $Y_{i}$ & $\hat{Y}_{i}$ & \\
\hline \multirow{7}{*}{0} & 1 & 95,25 & 0,00 & 95,25 & 95,25 & 99,00 & 101,95 & 94,00 & 106,09 & 89,00 & 108,42 & 91,75 & 109,56 & 92,25 & 109,04 & 96,50 & 104,23 & 95,65 \\
\hline & 2 & 42,75 & 0,00 & 42,75 & 42,75 & 44,50 & 51,23 & 46,50 & 58,84 & 46,50 & 65,39 & 48,00 & 75,19 & 48,50 & 78,59 & 43,00 & 84,83 & 79,71 \\
\hline & 3 & 25,00 & 0,00 & 25,00 & 25,00 & 24,50 & 24,70 & 25,25 & 25,33 & 24,75 & 26,57 & 25,25 & 29,98 & 24,50 & 31,80 & 25,50 & 37,79 & 39,85 \\
\hline & 4 & 14,75 & 0,00 & 14,75 & 14,75 & 13,50 & 13,56 & 12,75 & 12,80 & 14,00 & 12,43 & 15,50 & 12,63 & 15,75 & 13,06 & 13,75 & 15,49 & 17,93 \\
\hline & 5 & 7,25 & 0,00 & 7,25 & 7,25 & 8,00 & 8,74 & 9,25 & 9,56 & 8,25 & 10,00 & 7,75 & 10,49 & 8,00 & 10,73 & 9,75 & 12,28 & 15,82 \\
\hline & 6 & 4,00 & 0,00 & 4,00 & 4,00 & 3,75 & 2,95 & 3,25 & 2,51 & 3,25 & 2,34 & 4,00 & 2,28 & 3,75 & 2,30 & 2,25 & 2,55 & 3,46 \\
\hline & 7 & 6,00 & 0,00 & 6,00 & 6,00 & 6,25 & 5,01 & 6,50 & 4,16 & 5,50 & 3,49 & 5,00 & 2,63 & 5,25 & 2,38 & 5,00 & 1,99 & 2,65 \\
\hline \multirow{7}{*}{1} & 1 & 90,00 & 0,00 & 90,00 & 90,00 & 89,67 & 90,94 & 84,33 & 93,50 & 79,00 & 96,04 & 81,33 & 99,52 & 82,00 & 100,48 & 93,00 & 101,68 & 101,44 \\
\hline & 2 & 42,67 & 0,00 & 42,67 & 42,67 & 42,33 & 44,19 & 43,67 & 45,57 & 43,67 & 46,95 & 45,67 & 49,47 & 45,67 & 50,48 & 43,33 & 52,68 & 53,39 \\
\hline & 3 & 19,33 & 0,33 & 19,00 & 19,00 & 16,67 & 15,01 & 17,00 & 12,97 & 16,67 & 12,00 & 20,00 & 11,52 & 20,00 & 11,59 & 23,33 & 12,13 & 12,46 \\
\hline & 4 & 15,33 & 1,33 & 14,00 & 14,00 & 16,00 & 13,92 & 16,00 & 13,19 & 15,33 & 12,33 & 14,67 & 10,97 & 14,33 & 10,55 & 13,00 & 10,01 & 10,26 \\
\hline & 5 & 8,33 & 1,33 & 7,00 & 7,00 & 6,00 & 3,92 & 6,33 & 2,51 & 5,67 & 1,82 & 6,33 & 1,28 & 7,00 & 1,17 & 8,67 & 1,01 & 1,03 \\
\hline & 6 & 5,67 & 0,00 & 5,67 & 5,67 & 4,00 & 2,73 & 3,67 & 1,38 & 4,00 & 0,75 & 4,00 & 0,32 & 3,00 & 0,25 & 3,33 & 0,18 & 0,17 \\
\hline & 7 & 5,33 & 0,67 & 4,67 & 4,67 & 5,00 & 2,86 & 4,67 & 1,70 & 3,67 & 1,01 & 4,00 & 0,35 & 5,00 & 0,21 & 5,67 & 0,05 & 0,03 \\
\hline \multirow{7}{*}{2} & 1 & 95,00 & 0,00 & 95,00 & 95,00 & 97,33 & 97,99 & 94,67 & 100,73 & 92,67 & 103,64 & 90,67 & 106,64 & 77,67 & 107,51 & 80,00 & 108,26 & 107,44 \\
\hline & 2 & 35,67 & 0,00 & 35,00 & 35,00 & 35,00 & 40,21 & 36,00 & 47,85 & 37,33 & 47,85 & 40,00 & 52,19 & 37,67 & 53,39 & 46,33 & 54,62 & 53,72 \\
\hline & 3 & 19,67 & 0,33 & 20,00 & 20,00 & 20,00 & 21,41 & 21,00 & 24,67 & 20,00 & 24,67 & 20,33 & 26,89 & 20,00 & 27,55 & 20,00 & 28,26 & 27,79 \\
\hline & 4 & 11,33 & 0,33 & 11,00 & 11,00 & 10,00 & 11,14 & 9,33 & 11,60 & 10,33 & 11,60 & 11,00 & 12,02 & 9,00 & 12,15 & 11,00 & 12,26 & 12,11 \\
\hline & 5 & 7,67 & 1,00 & 6,67 & 6,67 & 8,00 & 8,21 & 8,00 & 8,96 & 7,67 & 8,96 & 7,00 & 8,81 & 7,00 & 8,70 & 7,00 & 8,46 & 8,48 \\
\hline & 6 & 5,00 & 0,00 & 5,00 & 5,00 & 4,00 & 3,93 & 4,00 & 3,47 & 4,33 & 3,47 & 4,33 & 3,28 & 4,00 & 3,21 & 3,33 & 3,08 & 3,13 \\
\hline & 7 & 5,67 & 0,67 & 5,00 & 5,00 & 5,00 & 3,67 & 4,67 & 2,07 & 4,33 & 2,07 & 5,00 & 1,44 & 4,33 & 1,31 & 4,33 & 1,27 & 1,40 \\
\hline \multirow{7}{*}{3} & 1 & 81,00 & 0,00 & 81,00 & 81,00 & 81,67 & 89,48 & 78,33 & 95,31 & 75,67 & 99,02 & 80,00 & 102,14 & 72,33 & 102,37 & 83,00 & 100,22 & 96,96 \\
\hline & 2 & 39,33 & 0,00 & 39,33 & 39,33 & 39,00 & 41,34 & 40,00 & 43,95 & 39,67 & 46,49 & 42,67 & 50,28 & 41,00 & 51,43 & 40,67 & 52,56 & 50,59 \\
\hline & 3 & 20,00 & 0,00 & 20,00 & 20,00 & 19,67 & 20,38 & 19,00 & 21,11 & 20,00 & 22,08 & 22,00 & 24,19 & 22,33 & 25,09 & 21,67 & 26,99 & 26,30 \\
\hline & 4 & 11,67 & 0,67 & 11,00 & 11,00 & 12,00 & 13,32 & 13,00 & 15,27 & 13,33 & 17,00 & 13,67 & 20,11 & 11,00 & 21,52 & 12,00 & 25,90 & 28,50 \\
\hline & 5 & 9,00 & 1,00 & 8,00 & 8,00 & 9,00 & 5,66 & 7,67 & 4,75 & 6,67 & 4,53 & 7,00 & 4,89 & 6,33 & 5,21 & 5,67 & 6,47 & 7,54 \\
\hline & 6 & 5,33 & 0,67 & 4,67 & 4,67 & 4,67 & 3,61 & 5,33 & 2,80 & 5,33 & 2,31 & 5,33 & 2,00 & 4,67 & 2,03 & 3,33 & 2,46 & 3,06 \\
\hline & 7 & 8,67 & 0,67 & 8,00 & 8,00 & 7,33 & 6,35 & 7,33 & 5,03 & 7,33 & 3,99 & 7,00 & 2,57 & 6,67 & 2,11 & 5,67 & 1,27 & 1,22 \\
\hline
\end{tabular}




\begin{tabular}{|c|c|c|c|c|c|c|c|c|c|c|c|c|c|c|c|c|c|c|}
\hline \multirow{2}{*}{ Trat } & \multirow{2}{*}{$\begin{array}{l}\mathscr{W} \\
\mathbb{0} \\
\mathbb{0} \\
\frac{\pi}{0}\end{array}$} & \multicolumn{4}{|c|}{1984} & \multicolumn{2}{|c|}{1986} & \multicolumn{2}{|c|}{1988} & \multicolumn{2}{|c|}{1990} & \multicolumn{2}{|c|}{1994} & \multicolumn{2}{|c|}{1996} & \multicolumn{2}{|c|}{2004} & \multirow{2}{*}{$\begin{array}{l}\text { 읗 } \\
\text { 言 } \\
\end{array}$} \\
\hline & & $Y_{i}$ & $h_{i}$ & $Y_{\text {if }}$ & $\hat{Y}_{i}$ & $Y_{i}$ & $\hat{Y}_{i}$ & $Y_{i}$ & $\hat{Y}_{i}$ & $Y_{i}$ & $\hat{Y}_{i}$ & $Y_{i}$ & $\hat{Y}_{i}$ & $Y_{i}$ & $\hat{Y}_{i}$ & $Y_{i}$ & $\hat{Y}_{i}$ & \\
\hline \multirow{7}{*}{4} & 1 & 92,67 & 0,00 & 92,67 & 92,67 & 92,00 & 93,08 & 90,00 & 93,86 & 85,00 & 94,48 & 82,00 & 94,79 & 80,33 & 94,75 & 90,67 & 94,44 & 94,38 \\
\hline & 2 & 44,00 & 0,00 & 44,00 & 44,00 & 42,00 & 42,65 & 42,33 & 41,91 & 44,00 & 41,56 & 46,00 & 41,33 & 46,00 & 41,28 & 47,00 & 41,13 & 41,03 \\
\hline & 3 & 23,67 & 0,33 & 23,33 & 23,33 & 21,67 & 14,21 & 22,67 & 22,15 & 23,00 & 22,49 & 24,00 & 22,01 & 21,67 & 21,88 & 22,67 & 21,63 & 21,49 \\
\hline & 4 & 11,67 & 0,00 & 11,67 & 11,67 & 12,67 & 2,97 & 13,00 & 15,94 & 13,33 & 17,09 & 13,00 & 18,34 & 12,33 & 18,62 & 11,67 & 19,02 & 18,79 \\
\hline & 5 & 4,33 & 1,00 & 3,33 & 3,33 & 4,67 & 2,97 & 4,33 & 2,96 & 4,33 & 3,08 & 5,67 & 3,35 & 4,67 & 3,46 & 7,33 & 3,65 & 3,67 \\
\hline & 6 & 3,67 & 1,00 & 2,67 & 2,67 & 2,00 & 1,77 & 3,00 & 1,43 & 3,33 & 1,34 & 2,67 & 1,39 & 3,00 & 1,34 & 1,67 & 1,54 & 1,56 \\
\hline & 7 & 5,33 & 0,67 & 4,67 & 4,67 & 3,33 & 2,19 & 2,67 & 1,16 & 2,67 & 0,73 & 3,33 & 0,52 & 3,00 & 0,51 & 3,67 & 0,55 & 0,56 \\
\hline \multirow{7}{*}{5} & 1 & 78,00 & 0,00 & 78,00 & 78,00 & 80,00 & 86,26 & 74,67 & 93,14 & 72,00 & 98,32 & 81,67 & 104,49 & 83,67 & 106,10 & 95,33 & 108,15 & 108,10 \\
\hline & 2 & 43,33 & 0,00 & 43,33 & 43,33 & 39,67 & 34,06 & 36,67 & 30,73 & 36,33 & 30,04 & 37,00 & 31,02 & 37,33 & 31,63 & 40,67 & 32,88 & 33,03 \\
\hline & 3 & 22,33 & 0,33 & 22,00 & 22,00 & 19,33 & 19,30 & 21,33 & 16,72 & 20,67 & 15,05 & 20,33 & 13,88 & 20,00 & 13,86 & 22,67 & 14,36 & 14,57 \\
\hline & 4 & 11,33 & 2,00 & 9,33 & 9,33 & 11,67 & 10,70 & 12,00 & 10,54 & 12,33 & 9,83 & 13,33 & 8,63 & 12,67 & 8,35 & 12,67 & 8,30 & 8,50 \\
\hline & 5 & 6,67 & 1,00 & 5,67 & 5,67 & 5,00 & 3,76 & 5,00 & 3,20 & 5,00 & 2,91 & 5,67 & 2,50 & 6,33 & 2,37 & 8,00 & 2,26 & 2,32 \\
\hline & 6 & 4,33 & 0,67 & 3,67 & 3,67 & 3,00 & 2,03 & 3,33 & 1,25 & 2,67 & 0,89 & 2,67 & 0,62 & 2,67 & 0,56 & 4,00 & 0,49 & 0,50 \\
\hline & 7 & 7,33 & 1,33 & 6,00 & 6,00 & 5,33 & 3,49 & 5,33 & 2,03 & 5,33 & 1,21 & 5,67 & 0,51 & 5,67 & 0,37 & 4,67 & 0,22 & 0,21 \\
\hline \multirow{7}{*}{6} & 1 & 76,33 & 0,00 & 76,33 & 76,33 & 75,67 & 83,77 & 73,33 & 89,53 & 71,67 & 93,50 & 72,67 & 97,33 & 70,33 & 98,40 & 90,67 & 99,02 & 98,75 \\
\hline & 2 & 42,33 & 0,00 & 42,33 & 42,33 & 39,00 & 39,61 & 40,67 & 39,09 & 40,33 & 39,61 & 45,33 & 41,32 & 40,33 & 42,04 & 40,67 & 43,35 & 43,45 \\
\hline & 3 & 17,67 & 0,67 & 17,00 & 17,00 & 18,33 & 17,27 & 18,67 & 17,14 & 17,67 & 17,10 & 18,67 & 17,45 & 19,00 & 17,74 & 19,00 & 18,58 & 18,83 \\
\hline & 4 & 13,33 & 0,67 & 12,67 & 12,67 & 10,67 & 9,36 & 11,00 & 7,74 & 11,00 & 6,94 & 9,33 & 6,41 & 9,00 & 6,38 & 11,00 & 6,63 & 6,80 \\
\hline & 5 & 4,67 & 2,00 & 2,67 & 2,67 & 4,33 & 2,26 & 4,00 & 1,85 & 3,67 & 1,56 & 4,67 & 1,27 & 3,67 & 1,23 & 4,33 & 1,22 & 1,26 \\
\hline & 6 & 5,67 & 0,00 & 5,67 & 5,67 & 3,67 & 3,13 & 3,67 & 1,98 & 4,33 & 1,40 & 4,00 & 0,92 & 3,67 & 0,83 & 2,67 & 0,75 & 0,78 \\
\hline & 7 & 5,67 & 2,33 & 3,33 & 3,33 & 3,33 & 1,62 & 3,33 & 0,82 & 3,33 & 0,44 & 3,67 & 0,18 & 4,00 & 0,14 & 4,33 & 0,10 & 0,10 \\
\hline \multirow{7}{*}{7} & 1 & 78,33 & 0,00 & 78,33 & 78,33 & 75,67 & 86,85 & 74,00 & 93,80 & 75,00 & 99,04 & 82,00 & 105,29 & 77,67 & 106,91 & 74,67 & 108,80 & 108,35 \\
\hline & 2 & 43,67 & 0,33 & 43,33 & 43,33 & 42,00 & 40,00 & 40,67 & 38,97 & 41,33 & 39,19 & 45,33 & 40,91 & 43,67 & 41,82 & 43,67 & 43,94 & 44,33 \\
\hline & 3 & 22,00 & 0,33 & 21,67 & 21,67 & 21,33 & 21,27 & 21,67 & 20,66 & 22,00 & 20,22 & 21,00 & 20,13 & 20,67 & 20,35 & 21,00 & 21,49 & 22,16 \\
\hline & 4 & 16,00 & 1,33 & 14,67 & 14,67 & 15,33 & 10,96 & 13,67 & 8,85 & 13,00 & 7,62 & 11,33 & 6,51 & 9,67 & 6,31 & 9,67 & 6,34 & 6,65 \\
\hline & 5 & 7,67 & 0,67 & 7,00 & 7,00 & 6,33 & 4,46 & 6,67 & 3,13 & 6,00 & 2,41 & 6,67 & 1,79 & 7,00 & 1,66 & 5,33 & 1,57 & 1,67 \\
\hline & 6 & 4,33 & 0,33 & 4,00 & 4,00 & 4,00 & 3,43 & 4,33 & 2,60 & 4,33 & 1,95 & 4,00 & 1,24 & 4,33 & 1,07 & 5,33 & 0,89 & 0,94 \\
\hline & 7 & 5,33 & 1,33 & 4,00 & 4,00 & 3,67 & 2,40 & 3,67 & 1,58 & 3,67 & 1,10 & 4,00 & 0,60 & 4,00 & 0,47 & 3,33 & 0,32 & 0,32 \\
\hline
\end{tabular}

$$
\bar{Y}=\left[\begin{array}{l}
Y_{1} \\
Y_{2} \\
Y_{3} \\
Y_{4} \\
Y_{5} \\
Y_{6} \\
Y_{7}
\end{array}\right]=\left[\begin{array}{r}
94,38 \\
41,03 \\
21,49 \\
18,97 \\
3,67 \\
1,56 \\
0,56
\end{array}\right]
$$

A solução geral do sistema foi obtida pela equação 19 . Determinaram-se os valores das constantes $\mathrm{c}_{1}$ a $\mathrm{c}_{7}$ substituindo os valores impostos pelas condiçóes iniciais do problema $\left(\boldsymbol{Y}_{i f}\right)$. Assume-se, por conveniência, que o tempo para as condiçôes iniciais do povoamento seja o momento zero, isto é, $\mathrm{t}_{0}=0$. Finalmente, o número de árvores em cada classe de DAP, para qualquer ponto no tempo.
Assim, a evolução da distribuição diamétrica, para cada tratamento, pode ser adequadamente formulada e resolvida como um sistema de equaçôes diferenciais de primeira ordem. Para cada classe de diâmetro é montada uma equação diferencial, que reflete a taxa líquida de variação no número de árvores, para o intervalo de tempo de 5,92 anos. Essa taxa líquida é a diferença algébrica entre o número de indivíduos ingressando em cada classe menos o número de indivíduos que deixaram essa mesma classe no intervalo de tempo considerado.

A solução do sistema de equações diferenciais conduz a equaçôes individuais, que descrevem o número de árvores em cada classe diamétrica como uma função do tempo. As situações de manejo (extração de madeira), representadas pelas quantidades de indivíduos removidos de cada classe de diâmetro $\left(\boldsymbol{b}_{i}\right)$ foram incorporadas ao modelo. 
Tabela 5 - Continuação ...

\begin{tabular}{|c|c|c|c|c|c|c|c|c|c|c|c|c|c|c|c|c|c|c|}
\hline \multirow{2}{*}{ Trat } & \multirow{2}{*}{$\begin{array}{l}\mathscr{D} \\
\mathbb{N} \\
\mathbb{D} \\
\frac{\mathbb{U}}{\mathrm{J}}\end{array}$} & \multicolumn{4}{|c|}{1984} & \multicolumn{2}{|c|}{1986} & \multicolumn{2}{|c|}{1988} & \multicolumn{2}{|c|}{1990} & \multicolumn{2}{|c|}{1994} & \multicolumn{2}{|c|}{1996} & \multicolumn{2}{|c|}{2004} & \multirow{2}{*}{$\begin{array}{l}\text { 음 } \\
\text { 言 } \\
\text { 戛 }\end{array}$} \\
\hline & & $Y_{i}$ & $h_{i}$ & $Y_{\text {if }}$ & $\hat{Y}_{i}$ & $Y_{i}$ & $\hat{Y}_{i}$ & $Y_{i}$ & $\hat{Y}_{i}$ & $Y_{i}$ & $\hat{Y}_{i}$ & $Y_{i}$ & $\hat{Y}_{i}$ & $Y_{i}$ & $\hat{Y}_{i}$ & $Y_{i}$ & $\hat{Y}_{i}$ & \\
\hline \multirow{7}{*}{8} & 1 & 80,33 & 0,00 & 80,33 & 80,33 & 78,33 & 90,72 & 75,33 & 99,82 & 75,67 & 106,98 & 79,00 & 116,09 & 71,67 & 118,70 & 80,00 & 122,81 & 123,31 \\
\hline & 2 & 37,67 & 0,00 & 37,67 & 37,67 & 34,33 & 30,97 & 33,33 & 27,83 & 34,33 & 26,69 & 35,33 & 26,93 & 33,00 & 27,44 & 33,67 & 29,01 & 29,60 \\
\hline & 3 & 19,00 & 0,33 & 18,67 & 18,67 & 17,00 & 14,77 & 18,00 & 11,95 & 16,33 & 10,06 & 18,33 & 8,20 & 18,00 & 7,84 & 20,00 & 7,71 & 7,97 \\
\hline & 4 & 13,33 & 1,00 & 12,33 & 12,33 & 11,33 & 9,74 & 11,67 & 7,72 & 11,67 & 6,21 & 11,67 & 4,39 & 9,33 & 3,90 & 7,00 & 3,30 & 3,38 \\
\hline & 5 & 8,33 & 1,00 & 7,33 & 7,33 & 7,00 & 4,78 & 6,00 & 3,33 & 6,00 & 2,44 & 7,00 & 1,48 & 6,33 & 1,23 & 8,67 & 0,86 & 0,85 \\
\hline & 6 & 6,67 & 1,67 & 5,00 & 5,00 & 5,33 & 2,77 & 5,33 & 1,67 & 4,67 & 1,09 & 4,67 & 0,57 & 5,00 & 0,45 & 4,67 & 0,26 & 0,25 \\
\hline & 7 & 6,33 & 1,67 & 4,67 & 4,67 & 3,00 & 2,71 & 3,67 & 1,58 & 4,67 & 0,95 & 5,33 & 0,41 & 4,33 & 0,29 & 3,67 & 0,14 & 0,12 \\
\hline \multirow{7}{*}{9} & 1 & 87,67 & 0,00 & 87,67 & 87,67 & 84,00 & 83,94 & 79,33 & 83,06 & 78,00 & 82,97 & 85,00 & 83,02 & 89,33 & 82,98 & 93,67 & 82,72 & 82,62 \\
\hline & 2 & 41,33 & 0,00 & 41,33 & 41,33 & 41,00 & 42,51 & 39,67 & 42,84 & 42,67 & 42,96 & 44,00 & 43,09 & 43,33 & 43,12 & 50,00 & 43,06 & 42,96 \\
\hline & 3 & 20,00 & 0,33 & 19,67 & 19,67 & 20,33 & 22,54 & 21,67 & 24,50 & 21,33 & 25,79 & 22,00 & 27,16 & 22,67 & 27,51 & 26,33 & 28,01 & 28,02 \\
\hline & 4 & 12,33 & 1,33 & 11,00 & 11,00 & 11,33 & 8,83 & 10,67 & 8,40 & 10,00 & 8,53 & 12,00 & 9,02 & 13,00 & 9,20 & 10,00 & 9,52 & 9,55 \\
\hline & 5 & 8,33 & 0,33 & 8,00 & 8,00 & 6,00 & 4,78 & 6,33 & 3,36 & 6,00 & 2,81 & 5,33 & 2,62 & 5,00 & 2,64 & 9,00 & 2,76 & 2,79 \\
\hline & 6 & 3,00 & 1,33 & 1,67 & 1,67 & 3,00 & 1,92 & 3,00 & 1,67 & 3,33 & 1,39 & 4,33 & 1,08 & 4,33 & 1,02 & 2,33 & 1,00 & 1,01 \\
\hline & 7 & 6,67 & 1,33 & 5,33 & 5,33 & 3,33 & 2,22 & 3,33 & 1,07 & 3,33 & 0,60 & 3,67 & 0,30 & 3,33 & 0,26 & 4,67 & 0,22 & 0,22 \\
\hline \multirow{7}{*}{10} & 1 & 82,67 & 0,00 & 82,67 & 82,67 & 77,00 & 86,36 & 75,00 & 89,85 & 72,67 & 92,40 & 82,67 & 95,32 & 71,00 & 96,10 & 87,00 & 97,24 & 97,25 \\
\hline & 2 & 34,00 & 0,00 & 34,00 & 34,00 & 31,67 & 33,94 & 31,33 & 34,48 & 34,00 & 35,29 & 36,00 & 36,82 & 33,00 & 37,41 & 34,33 & 38,61 & 38,90 \\
\hline & 3 & 21,67 & 0,33 & 21,33 & 21,33 & 20,67 & 18,21 & 19,67 & 16,15 & 20,00 & 14,86 & 20,00 & 13,65 & 20,00 & 13,44 & 19,33 & 13,41 & 13,61 \\
\hline & 4 & 11,67 & 0,33 & 11,33 & 11,33 & 10,67 & 10,46 & 12,33 & 9,43 & 11,33 & 8,51 & 12,33 & 7,28 & 12,00 & 6,94 & 10,00 & 6,51 & 6,59 \\
\hline & 5 & 5,33 & 2,33 & 3,00 & 3,00 & 5,67 & 4,04 & 5,00 & 4,30 & 5,67 & 4,19 & 5,00 & 3,68 & 4,33 & 3,46 & 6,33 & 3,04 & 3,03 \\
\hline & 6 & 4,33 & 0,00 & 4,33 & 4,33 & 1,67 & 1,31 & 1,33 & 0,60 & 1,33 & 0,42 & 1,67 & 0,33 & 2,33 & 0,31 & 2,33 & 0,26 & 0,26 \\
\hline & 7 & 7,67 & 3,00 & 4,67 & 4,67 & 3,67 & 1,58 & 4,00 & 0,55 & 3,33 & 0,20 & 4,00 & 0,06 & 3,33 & 0,05 & 2,67 & 0,04 & 0,03 \\
\hline \multirow{7}{*}{11} & 1 & 84,00 & 0,00 & 84,00 & 84,00 & 85,67 & 79,49 & 79,00 & 78,95 & 72,67 & 79,43 & 75,67 & 80,44 & 76,67 & 80,70 & 102,67 & 80,96 & 80,94 \\
\hline & 2 & 32,00 & 0,00 & 32,00 & 32,00 & 29,33 & 31,97 & 31,00 & 31,56 & 33,33 & 31,40 & 39,33 & 31,57 & 36,67 & 31,70 & 40,00 & 31,94 & 31,95 \\
\hline & 3 & 24,00 & 2,00 & 22,00 & 22,00 & 22,33 & 21,88 & 23,00 & 21,70 & 23,33 & 21,52 & 21,33 & 21,38 & 21,00 & 21,41 & 22,33 & 21,59 & 21,64 \\
\hline & 4 & 15,33 & 2,00 & 13,33 & 13,33 & 14,00 & 7,66 & 12,33 & 5,83 & 9,67 & 5,21 & 10,33 & 4,91 & 10,67 & 4,89 & 9,67 & 4,92 & 4,94 \\
\hline & 5 & 8,33 & 1,33 & 7,00 & 7,00 & 6,00 & 6,06 & 8,00 & 4,68 & 8,33 & 3,75 & 7,33 & 2,94 & 6,33 & 2,81 & 9,00 & 2,70 & 2,71 \\
\hline & 6 & 4,00 & 1,33 & 2,67 & 2,67 & 3,00 & 1,14 & 2,67 & 0,68 & 1,67 & 0,49 & 2,67 & 0,34 & 2,67 & 0,31 & 2,33 & 0,29 & 0,29 \\
\hline & 7 & 5,00 & 1,00 & 4,00 & 4,00 & 2,67 & 1,35 & 2,67 & 0,48 & 2,33 & 0,20 & 2,00 & 0,06 & 2,33 & 0,05 & 1,33 & 0,04 & 0,04 \\
\hline \multirow{7}{*}{12} & 1 & 79,67 & 0,00 & 79,67 & 79,67 & 74,00 & 80,33 & 69,67 & 82,15 & 73,67 & 83,96 & 82,67 & 86,60 & 86,00 & 87,42 & 96,00 & 88,76 & 88,84 \\
\hline & 2 & 38,33 & 0,33 & 38,00 & 38,00 & 37,33 & 36,38 & 37,33 & 35,72 & 36,67 & 35,69 & 38,33 & 36,37 & 36,67 & 36,78 & 43,67 & 37,80 & 38,07 \\
\hline & 3 & 21,67 & 0,33 & 21,33 & 21,33 & 19,00 & 16,17 & 18,33 & 13,31 & 18,33 & 11,76 & 16,67 & 10,59 & 15,00 & 10,45 & 18,67 & 10,56 & 10,71 \\
\hline & 4 & 13,67 & 1,00 & 12,67 & 12,67 & 14,00 & 12,46 & 15,33 & 11,29 & 14,00 & 10,02 & 15,00 & 8,22 & 15,33 & 7,71 & 14,33 & 7,08 & 7,14 \\
\hline & 5 & 7,67 & 1,67 & 6,00 & 6,00 & 6,67 & 5,14 & 7,00 & 4,61 & 6,33 & 4,13 & 6,67 & 3,32 & 6,00 & 3,04 & 7,00 & 2,59 & 2,58 \\
\hline & 6 & 5,67 & 1,00 & 4,67 & 4,67 & 3,33 & 3,02 & 3,33 & 2,25 & 4,33 & 1,83 & 5,67 & 1,38 & 5,67 & 1,23 & 4,00 & 0,96 & 0,93 \\
\hline & 7 & 4,67 & 2,33 & 2,33 & 2,33 & 1,33 & 0,79 & 1,67 & 0,35 & 1,67 & 0,21 & 1,67 & 0,13 & 1,67 & 0,11 & 2,67 & 0,08 & 0,08 \\
\hline
\end{tabular}

Como observado, o sistema de equações diferenciais de primeira ordem mantém a simplicidade de abordagem dos problemas inerente aos modelos baseados em matrizes de transição, apresentando a vantagem adicional de permitir a obtenção dos valores das variáveis do sistema em qualquer ponto no tempo, não se restringindo a intervalos múltiplos do intervalo original usado na derivação da matriz de transição.

Este estudo fez coincidir os períodos de projeção com as observaçōes reais de campo (1984, 1986, 1988, 1990, 1994, 1996 e 2004). Na Tabela 5 são apresentados os valores observados e estimados com o uso do modelo para cada tratamento.

Observa-se na tabela que todos os tratamentos apresentam um ponto de equilíbrio. Como todo autovalor real de $\mathbf{A}$ e todo autovalor complexo de A tem parte real negativa, para cada tratamento, o ponto de equilíbrio é assintoticamente estável (Zill \& Cullen, 2001). A tradicional forma de J invertido para distribuição dos diâmetros, neste ponto, é mantida.

Os valores preditos, $\hat{Y}_{i}$ sumarizados na Tabela 5 
Tabela 6 - Número de árvores por hectare e por tratamento, aneladas, que morreram em 1996 e 2004.

\begin{tabular}{|c|c|c|c|c|c|c|c|c|c|}
\hline \multirow{2}{*}{ CLASSEDAP } & \multicolumn{9}{|c|}{ TRATAMENTOS - n.ha-1 } \\
\hline & 2 & 3 & 4 & 6 & 7 & 8 & 10 & 11 & 12 \\
\hline \multicolumn{10}{|c|}{ ÁRVORES MORTAS EM 1996} \\
\hline 1 & 10,67 & 10,33 & 1,00 & 7,33 & 1,33 & 13,33 & 16,33 & 4,33 & 3,67 \\
\hline 2 & 3,00 & 2,67 & 3,33 & 5,00 & 1,00 & 3,67 & 3,67 & 2,67 & 2,00 \\
\hline 3 & 1,00 & 0,33 & 1,33 & 2,00 & 1,33 & 1,00 & 0,67 & 0,67 & 0,67 \\
\hline 4 & 1,00 & 1,00 & 1,00 & 0,33 & 1,00 & 1,33 & 0,67 & 0,00 & 0,67 \\
\hline 5 & 0,67 & 0,33 & 0,00 & 1,00 & 0,00 & 0,33 & 0,00 & 0,33 & 0,33 \\
\hline 6 & 0,33 & 0,67 & 0,33 & 0,00 & 0,33 & 0,00 & 0,00 & 0,33 & 0,00 \\
\hline 7 & 0,33 & 0,00 & 0,00 & 0,00 & 0,00 & 1,00 & 0,33 & 0,33 & 0,00 \\
\hline \multicolumn{10}{|c|}{ ÁRVORES MORTAS EM 2004} \\
\hline 1 & 0,33 & 1,00 & - & 0,67 & 0,67 & 0,33 & 1,67 & 0,33 & 0,33 \\
\hline 2 & 0,00 & 0,67 & - & 0,00 & 0,00 & 0,33 & 0,33 & 0,33 & 0,67 \\
\hline 3 & 0,33 & 0,67 & - & 0,00 & 0,33 & 0,33 & 0,00 & 0,00 & 0,33 \\
\hline 4 & 0,33 & 0,67 & - & 0,00 & 0,00 & 0,00 & 0,33 & 0,00 & 0,00 \\
\hline 5 & 0,67 & 0,33 & - & 0,00 & 0,00 & 0,00 & 0,00 & 0,00 & 0,00 \\
\hline 6 & 0,00 & 1,00 & - & 0,00 & 0,00 & 0,00 & 0,00 & 0,00 & 0,00 \\
\hline 7 & 0,00 & 0,33 & - & 0,00 & 0,33 & 0,00 & 0,00 & 0,00 & 0,00 \\
\hline
\end{tabular}

representam a capacidade do sistema de equações de estimar futuros estados do povoamento

Para os valores preditos ocorrem discrepâncias com o aumento no decorrer do tempo. Isto ocorre em função, primeiro, do ingresso e do crescimento de seguir rigorosamente as condições iniciais observadas e, segundo, as discrepâncias resultantes da mortalidade de árvores. É óbvio que a mortalidade em função dos primeiros 6 anos, devido a extração madeireira, foi muito maior que nos 14 anos remanescentes.

O sistema de equações diferenciais estima com acurácia valores para o número de árvores nas projeções feitas até os anos 4 (1988) a 6 (1990), entretanto, afasta-se da realidade, principalmente, para as projeçôes realizadas para 20 anos (2004). Ocorrem estimativas reais para classes intermediárias de diâmetros e também para determinado ano e tratamento.

Por este motivo evitaram-se projeçōes ao longo prazo que, em muitas circunstâncias, não são realísticas e introduzem muita subjetividade, além do que, dificilmente são comparadas aos dados reais.

A principal causa do decréscimo da acurácia com o passar do tempo é inerente à natureza do modelo. Outras importantes origens dos erros estão no intervalo de tempo (aproximadamente 6 anos) para estimar eventos tais como a mortalidade de grandes árvores, o qual ocorre esporadicamente e provavelmente não aleatoriamente no tempo e no espaço e na inclusão ou exclusão de perturbações.

Ek (1974) e Moser (1972) também tiveram dificuldades com projeçōes de longo prazo (20 anos), principalmente nas classes de maior tamanho. As projeções de longo prazo acumulam facilmente mais erros.

Este ceticismo não necessariamente invalida todas as predições, pelo contrário, isto indica a necessidade de ficar atento a possíveis origens de erros nos parâmetros usados no modelo, derivados da matriz de transição, e para incorporação de informações adicionais no processo de predição, como por exemplo, redução do número de árvores mortas devido aos tratamentos silviculturais.

A exatidão das predições sobre vários períodos depende de uma boa estimativa, que, por sua vez, depende da suficiência de dados para todas as classes de diâmetro (Bruner \& Moser, 1973).

Os sistemas de equações foram desenvolvidos de uma série histórica de dados sujeita a treze diferentes tratamentos, que foi

Tabela 7 - Número de árvores estimado por hectare e por classe de diâmetro para 0 tratamento 4 , corrigido.

\begin{tabular}{|c|c|c|c|c|c|c|c|c|}
\hline \multirow[b]{2}{*}{ 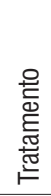 } & \multirow[b]{2}{*}{ 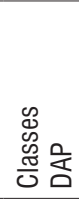 } & \multicolumn{3}{|c|}{1996} & \multicolumn{3}{|c|}{2004} & \multirow[b]{2}{*}{$\begin{array}{l}\text { 은 } \\
\text { 言 } \\
\text { 岀 }\end{array}$} \\
\hline & & $Y_{i}$ & $\hat{Y}_{i}$ & $\begin{array}{l}\text { 음 } \\
\text { 흥 } \\
\text { ¿ }\end{array}$ & $Y_{i}$ & $\hat{Y}_{i}$ & $\begin{array}{l}\text { 음 } \\
\text { 홍 } \\
0 \\
\text { - }\end{array}$ & \\
\hline \multirow{7}{*}{4} & 1 & 80,33 & 94,75 & 93,75 & 90,67 & 94,44 & 94,44 & 94,38 \\
\hline & 2 & 46,00 & 41,28 & 33,95 & 47,00 & 41,13 & 41,13 & 41,03 \\
\hline & 3 & 21,67 & 21,88 & 20,55 & 22,67 & 21,63 & 21,63 & 21,49 \\
\hline & 4 & 12,33 & 18,62 & 17,69 & 11,67 & 19,02 & 19,02 & 18,79 \\
\hline & 5 & 4,67 & 3,46 & 3,46 & 7,33 & 3,65 & 3,65 & 3,67 \\
\hline & 6 & 3,00 & 1,34 & 1,10 & 1,67 & 1,54 & 1,54 & 1,56 \\
\hline & 7 & 3,00 & 0,51 & 0,52 & 3,67 & 0,55 & 0,55 & 0,56 \\
\hline
\end{tabular}


Tabela 8 - Incremento periódico anual médio em diâmetro (cm ano-1), por tratamento, verificado nos períodos de medições sucessivas (1984 a 2004).

\begin{tabular}{|c|c|c|c|c|c|c|c|c|c|c|c|c|c|}
\hline \multirow{2}{*}{$\begin{array}{l}\text { Classe } \\
\text { DAP }\end{array}$} & \multicolumn{13}{|c|}{ TRATAMENTOS } \\
\hline & 0 & 1 & 2 & 3 & 4 & 5 & 6 & 7 & 8 & 9 & 10 & 11 & 12 \\
\hline 1 & 0,17 & 0,21 & 0,25 & 0,28 & 0,23 & 0,26 & 0,29 & 0,28 & 0,27 & 0,28 & 0,29 & 0,27 & 0,29 \\
\hline 2 & 0,25 & 0,28 & 0,34 & 0,35 & 0,31 & 0,32 & 0,34 & 0,33 & 0,34 & 0,34 & 0,37 & 0,37 & 0,37 \\
\hline 3 & 0,25 & 0,35 & 0,38 & 0,34 & 0,29 & 0,33 & 0,38 & 0,31 & 0,35 & 0,42 & 0,34 & 0,33 & 0,42 \\
\hline 4 & 0,26 & 0,35 & 0,40 & 0,36 & 0,36 & 0,41 & 0,34 & 0,39 & 0,35 & 0,45 & 0,42 & 0,36 & 0,39 \\
\hline 5 & 0,27 & 0,38 & 0,38 & 0,39 & 0,36 & 0,42 & 0,45 & 0,44 & 0,38 & 0,33 & 0,39 & 0,43 & 0,47 \\
\hline 6 & 0,28 & 0,31 & 0,28 & 0,33 & 0,34 & 0,49 & 0,34 & 0,35 & 0,39 & 0,41 & 0,46 & 0,59 & 0,41 \\
\hline 7 & 0,27 & 0,37 & 0,26 & 0,22 & 0,32 & 0,34 & 0,48 & 0,43 & 0,59 & 0,30 & 0,36 & 0,50 & 0,30 \\
\hline Média & 0,25 & 0,32 & 0,33 & 0,33 & 0,32 & 0,37 & 0,37 & 0,36 & 0,38 & 0,36 & 0,37 & 0,41 & 0,38 \\
\hline
\end{tabular}

desde a floresta sem interferência a diferentes níveis de redução de área basal, que constituiu os outros tratamentos.

Os tratamentos silviculturais aplicados em 1994 que causaram a morte de árvores em 1996 e 2004 (Tabela 6), não foram considerados na formulação do modelo. Uma forma de ajustar os dados estimados aos dados observados em 1996 e 2004 é remover dos valores estimados a fração de árvores mortas em conseqüência da aplicação do refinamento/ liberação.

$\mathrm{Na}$ Tabela 7 é mostrada a correção no número de árvores para o Tratamento 4. A colheita de madeira foi facilmente introduzida no modelo reavaliando a condição inicial do sistema no tempo $\mathrm{t}_{0}$. Entretanto, para os tratamentos silviculturais, o povoamento remanescente necessita de um intervalo de tempo para assimilar fotossinteticamente e utilizar completamente os resultados de aumento na energia solar, nutriente e água.

Inspecionando ainda os resultados na Tabela 5, um detalhe importante é a flutuação na densidade de árvores, o que poderia induzir a conclusões erradas, dependendo dos anos de medições que se tiver em mãos, sobre os processos dinâmicos que estariam ocorrendo na floresta. Este fato demonstra a importância de intervalos curtos entre medições e de períodos longos para estudo sobre dinâmica da floresta.

O número de árvores por hectare para cada período de observação e para as projeções, segue a forma de J invertido, com a freqüência diferindo uma da outra na menor classe de diâmetro. Este valor está sobre-estimado e na maior classe ocorre sub-estimativa da freqüência. Em particular acreditase que o aumento ocorrido no número de árvores na menor classe de diâmetro se deva à grande dificuldade em quantificar o ingresso.

De acordo com Valentine \& Furnival (1989), as formulaçôes com ingresso aumentam a exatidão e o realismo das projeções em curto prazo e fortalecem projeçôes intermediárias e em longo prazo, para matrizes de transição.

As projeções realizadas para os períodos superiores a 1990 também podem ser vistas como uma validação do modelo e como um indicador quantitativo de que o modelo é adequado aos dados.

Salomon et al. (1986) sugeriram a diferença média entre o valor atual e o valor predito, bem como o erro padrão da diferença média, como uma maneira de testar a exatidão do modelo. Neste estudo, o objetivo é comparar os números em termos absolutos e aceitar ou rejeitar o modelo para futuras projeções.

Até 2004 (Tabela 5), não houve reposição das árvores nas classes maiores. Aparentemente, as classes menores foram mais beneficiadas. Entretanto, não se pode afirmar que a aplicação dos tratamentos estimulou o crescimento em diâmetro do povoamento remanescente, pois o mesmo comportamento foi observado no tratamento testemunha. Nas análises estatísticas conclui-se que o aumento se deu em função do tempo e não da aplicação dos tratamentos. Autores como Gutierrez (1970) e De Graaf (1986) admitem que a intensidade de corte parece ter um efeito diferencial sobre o crescimento diamétrico nas diferentes classes.

Os resultados da Tabela 8 revelam que, de modo geral, a aplicação dos tratamentos estimulou o crescimento em diâmetro do povoamento remanescente, quando comparado ao Tratamento T0 (testemunha). Os resultados da Tabela 8 demonstram também que a hierarquia encontrada para o incremento médio anual não é a mesma seguida para a redução em área basal, isto é, o tratamento que sofreu o maior nível de interferência em área basal não foi o que apresentou a maior taxa de incremento médio anual.

A distribuição de diâmetros observada é muito semelhante à projetada (Tabela 5), o que parece confirmar o uso da equação de ingresso, para curtos períodos de projeção. Entretanto, mais estudos são necessários para predizer o comportamento do ingresso por longos períodos de tempo. $\mathrm{O}$ modelo parece adequado para estudar os efeitos de diferentes tratamentos ou opções de manejo (regimes de corte) nas taxas de crescimento.

Em geral, os resultados das projeçôes (Tabela 5) evidenciam que nem os períodos de projeções mais longos, ponto de 
equilíbrio, serão suficientes para restaurar as condiçōes iniciais do povoamento. Isto indica que a aplicação de cortes, por si só, conduz o povoamento para alguma situação diferente da situação inicial.

Em florestas tropicais, como no caso da área de estudo, os modelos estocásticos são mais confiáveis porque o comportamento da dinâmica florestal é muito mais probabilístico do que determinístico.

\section{CONCLUSÕES}

Com base nos resultados obtidos conclui-se que:

O sistema de equaçôes diferenciais estima o número de árvores com realismo para projeções em curto prazo, mas afasta-se da realidade para projeçôes em longo prazo;

A aplicação dos tratamentos, quando comparado ao Tratamento T0 (testemunha), estimulou o crescimento em diâmetro do povoamento remanescente;

A hierarquia encontrada para o incremento médio anual não é a mesma seguida para redução em área basal;

O sistema de equações diferenciais parece adequado para estudar os efeitos de diferentes tratamentos ou opções de manejo;

Em geral, os resultados das projeções com auxílio do sistema de equações diferenciais evidenciam que nem os períodos de projeçôes mais longos (ponto de equilíbrio) serão suficientes para restaurar as condiçôes iniciais do povoamento.

\section{AGRADECIMENTOS}

Ao Grupo Orsa, pelo apoio logístico na coleta dos dados. A Fundação de Amparo à Pesquisa do Estado do Amazonas - FAPEAM, pela bolsa de estudo.

\section{REFERÊNCIAS BIBLIOGRÁFICAS}

Alder, D. 1995 Growth Modelling for Mixed Tropical Forests. Department of Plant Sciences, University of Oxford, Tropical Forestry Paper 30. 231pp.

Azevedo, C.P. 1993. Predição da distribuiçāo diamétrica de povoamentos florestais inequiâneos pelo emprego da matriz de transição. Dissertação de Mestrado. Universidade Federal de Viçosa, Viçosa, MG. 118pp.

Botkin, D.B.; Janak, J.F.; Wallis, J.R. 1972. Some ecological consequences of a computer model of forest growth. Journal of Ecology, 60:849-72.

Bruner, H.D.; Moser Jr, J.W. 1973. A Markov Chain Approach to the Prediction of Diameter Distributions in Uneven-aged Forest Stands. Canadian Journal of Forest Research, 3:409-417.

Buongiorno, J.; Michie, B.R. 1980. A matrix model of uneven-aged forest management. Forest Science, 26: 609-25.
Clutter, J.L. 1963. Compatible growth and yield models for lobolly pine. Forest Science, 9: 354-71.

Daniels, R.F.; Burkhart H.E. 1988. An integrated system of forest stand models. Forest Ecology and Management, 23: 159-77.

Davidson, C. B.; Halfley, W. L.; Frederick, D. J.; Mengel, D. L. 1989. Prediction of total stand ingrowth in southeastern mixed species bottomland hardwoods. In Miller, J.H. comp. Fifth biennial southern silvicultural research conference. New Orleans, Louisiana, Southern Forest Experiment Station, p. 435-9

De Graaf, N.R. 1986. A silvicultural system for natural regeneration of tropical rainforest in Suriname. Wageningem, The Netherlands: University of Wageningem. 250pp.

Ek, A.R. 1974. Nonlinear models for stand table projection in northeern hardwood. Canadian Journal of Forest Research, 4: 23-7.

Gutierrez, A.M.R. 1970. Efecto del raleo sobre el crescimiento en area basal de un bosque secundario en el tropico humedo. Turrialba. Dissertação de Mestrado. Instituto Interamericano de Ciencias Agricolas de la OEA, Costa Rica. 79pp.

Higuchi, N. 1987. Short-Term growth of an undisturbed tropical moist forest in the Brazilian Amazon. PhD Thesis. Michigan State University. 129pp.

Moser Jr, J.W. 1972. Dynamics of an uneven-aged forest stand. Forest Science, 18: 184-191.

Ribeiro, C.A.A.S. 1996. Um modelo para projeção da distribuição diamétrica de povoamentos inequiâneos baseados em equaçôes diferenciais. Revista Árvore, 20(3): 279 - 286.

Sanquetta, C.R.; Brena, D.A.; Angelo H.; Mendes J.B. 1996. Matriz de transição para simulação da dinâmica de florestas naturais sob diferentes intensidades de corte. Revista Floresta, 6(1): 65-78.

Silva, J.N.M.; Lopes, J.C.A. 1984. Inventário florestal contínuo em florestas tropicais: a metodologia utilizada pela EMBRAPA-CPATU na Amazônia brasileira. Belém: Embrapa-CPATU, 36pp.

Solomon, D. S.; Hosmer, R. A.; Hayslett JR., H. T. 1986. A forest two-stage matrix model for predicting growth of forest stands in the northeast. Canadian Journal of Forest Research. 16:521508 .

Sullivan, A.D.; Clutter, J. L. 1972. A simultaneous growth and yield model for loblolly pine. Forest Science. 18: 76-86.

Valentine, H. T.; Furnival, G. M. 1989. Projections with ingrowth by Markov chains. Forest Science, 35: 245-50.

Vanclay, J.K. 1989. A growth model for North Queensland rainforests. Forest Ecology and Management. 27: 245-271.

Zill, D. G. 2003. Equaçôes diferenciais com aplicaçōes em modelagem. São Paulo. Pioneira Thomson Learning.

Zill, D.G.; Cullen, M.R. 2001. Equaçôes diferenciais - Volume 2. São Paulo. Makron Books.

Recebido em 19/04/2007

Aceito em 12/09/2007 